Gao and Johnson et al.

\title{
Structural and biochemical analyses of selectivity determinants in chimeric Streptococcus Class A sortase enzymes
}

by

Melody Gao, ${ }^{1 *}$ D. Alex Johnson, ${ }^{1 \$}$ Isabel M. Piper, ${ }^{1}$ Hanna M. Kodama, ${ }^{1}$ Justin E. Svendsen, ${ }^{1}$ Elise Tahti, ${ }^{1}$ Brandon Vogel, ${ }^{1}$ John M. Antos, ${ }^{1}$ Jeanine F. Amacher ${ }^{1}{ }^{*}$

${ }^{1}$ Department of Chemistry, Western Washington University, Bellingham, WA, 98225

These authors contributed equally to this work.

*Corresponding Author:

Jeanine Amacher, Department of Chemistry, Western Washington University, 516 High St MS9150, Bellingham, WA, 98225, Tel: +1-360-650-4397, Fax: +1-360-650-2826

Email: amachej@wwu.edu

Running title: Selectivity determinants of Streptococcus Class A sortases

Keywords: sortases, enzymes, target selectivity, structural biology, protein biochemistry, protein engineering 
Gao and Johnson et al.

\begin{abstract}
Sequence variation in related proteins is an important characteristic that modulates activity and selectivity. An example of a protein family with a large degree of sequence variation is that of bacterial sortases, which are cysteine transpeptidases on the surface of gram-positive bacteria. Class A sortases are responsible for attachment of diverse proteins to the cell wall to facilitate environmental adaption and interaction. These enzymes are also used in protein engineering applications for sortase-mediated ligations (SML) or sortagging of protein targets. We previously investigated SrtA from Streptococcus pneumoniae, identifying a number of putative $\beta 7-\beta 8$ loop-mediated interactions that affected in vitro enzyme function. We identified residues that contributed to the ability of $S$. pneumoniae SrtA to recognize several amino acids at the P1' position of the substrate motif, underlined in LPXT $\underline{\text {, }}$, in contrast to the strict P1' Gly recognition of SrtA from Staphylococcus aureus. However, motivated by the lack of a structural model for the active, monomeric form of $S$. pneumoniae SrtA, here, we expanded our studies to other Streptococcus SrtA proteins. We solved the first monomeric structure of S. agalactiae SrtA which includes the C-terminus, and three others of $\beta 7-\beta 8$ loop chimeras from S.pyogenes and S. agalactiae SrtA. These structures and accompanying biochemical data support our previously identified $\beta 7-\beta 8$ loop-mediated interactions and provide additional insight into their role in Class A sortase substrate selectivity. We argue that a greater understanding of individual SrtA sequence and structural determinants of target selectivity can facilitate the design or discovery of improved sortagging tools.
\end{abstract}


Gao and Johnson et al.

\section{Introduction}

Class A sortases are enzymes located on the surface of gram-positive bacteria that attach proteins to the cell wall. Sortase-mediated protein display allows bacteria to interact with their environments, e.g., with proteins for bacterial adhesion and/or acquisition of nutrients, and can include pathogenic factors that enable the bacteria to infect host organisms. ${ }^{1,2}$ The catalytic mechanism of sortases involves the recognition and cleavage of a specific sequence, followed by ligation of an incoming amine nucleophile. ${ }^{1-3}$ This reactivity has also been harnessed for protein engineering applications, and sortases have emerged as powerful tools for the post-translational derivatization of protein targets with various non-native modifications. ${ }^{3}$ The traditional recognition motif of Class A sortase (or SrtA) proteins, which is found within the cell wall sorting signal (CWSS) of gram positive bacteria, is the sequence LPXTG (where $\mathrm{X}=$ any amino acid, and $\mathrm{L}=\mathrm{P} 4, \mathrm{P}=\mathrm{P} 3, \mathrm{X}=\mathrm{P} 2, \mathrm{~T}=\mathrm{P} 1$, and $\mathrm{G}=\mathrm{P} 1^{\prime}$ ). This sequence is recognized by all Class $\mathrm{A}$ sortases investigated to date, however, other recognition sequences have been identified or engineered for several SrtA proteins in the last decade, greatly increasing the potential for sortase-mediated ligation (SML), or sortagging, applications. ${ }^{4-12}$

Despite a relatively large degree of sequence variation amongst the hundreds of identified SrtA proteins in bacteria, these cysteine transpeptidases contain a conserved catalytic triad, consisting of His, Cys, and Arg residues. ${ }^{1,2,13}$ The most well studied Class A sortase is that from Staphylococcus aureus (saSrtA), which continues to see frequent use in sortagging applications. ${ }^{3}$ As of 2019, there were approximately 10 known structures of Class A sortases, with several being of saSrtA. ${ }^{13}$ Overall, the sortase fold, consisting of a closed 8 -stranded $\beta$-barrel architecture, is conserved in all structures of SrtA proteins solved to date; however, there are variations consistent with the degree of sequence differences. ${ }^{2}$ For example, between saSrtA and Streptococcus 
Gao and Johnson et al.

pyogenes SrtA (spySrtA), there are a number of unique structural characteristics that affect enzyme function (Figure 1). Specifically, saSrtA requires a $\mathrm{Ca}^{2+}$ cofactor and its $\beta 7-\beta 8$ loop near the active site contains an additional 5 residues and a Trp (W194) which dramatically affects activity (Figure 1a). ${ }^{12,14}$ All structural comparisons with saSrtA will use the peptidomimetic-bound structure (PDB ID 2 KID) as this is the only one to our knowledge of saSrtA in the active state. ${ }^{15-19}$ Previous work shows that allosteric activation, driven by $\mathrm{Ca}^{2+}$ binding, affects several structural features near the active site, including the relative conformation and/or location of the $\beta 6-\beta 7$ and $\beta 7-\beta 8$ loops. ${ }^{15-20}$ In the case of spySrtA, a partially helical C-terminal extension of 24 residues is evident in the reported crystal structure that is absent in SaSrtA (Figure 1). A detailed description of how each of these features determine target recognition and selectivity remains incomplete, particularly for the Streptococcus SrtA proteins. Unique features of B. anthracis SrtA (baSrtA) have also been previously described, e.g., regulation of enzymatic activity by an $\mathrm{N}$-terminal appendage as well as a disordered-to-ordered transition in the $\beta 7-\beta 8$ loop upon ligand binding. ${ }^{21}$

A number of protein families use specificity-determining loops to encode differing target selectivity amongst members. Classic examples include kinases and serine proteases. ${ }^{22-28}$ Specific regions of the activation loop of kinases contribute to substrate specificity by directly interacting with amino acids adjacent to the phosphorylation site. ${ }^{25,28}$ In serine proteases, substitution of two conserved surface loops (9 residues total) efficiently converts selectivity of trypsin to that of the related enzyme chymotrypsin..$^{22,23}$ There are also examples in scaffolding domains, including SH2 and SH3 domains, where conserved loops interact directly with the peptide and determine the selectivity of both SH2 (the EF and BG loops) and SH3 (the RT and n-Src loops) domains. ${ }^{29-37}$ Work from ourselves and others strongly indicates that Class A sortases are another protein family that exhibits functionally relevant sequence variation in specificity-determining loops. ${ }^{12,38,39}$ 
Gao and Johnson et al.

In our previous work, we investigated the selectivity determinants of Streptococcus pneumoniae SrtA (spSrtA) at the P1' position of the CWSS. ${ }^{12}$ We found that the sequence of the $\beta 7-\beta 8$ loop dramatically affects enzyme activity and selectivity at this substrate position. ${ }^{12}$ Because spSrtA crystallizes as a domain-swapped dimer, which is enzymatically inactive in our hands, we used previously published Class A sortase structures to investigate the stereochemistry of our biochemical results. ${ }^{12,40,41}$ Now, we investigate two additional sortases, those from $S$. pyogenes (spySrtA) and $S$. agalactiae (sagSrtA), to see if the $\beta 7-\beta 8$ loop has broad effects on enzyme function and target recognition for Streptococcus Class A sortases.

We find that the $\beta 7-\beta 8$ loop affects spySrtA and sagSrtA in a manner consistent with that of $S$. pneumoniae SrtA. To investigate how the $\beta 7-\beta 8$ loop sequence affects each protein, we created a series of chimeric enzymes, swapping the loop sequences from several of those previously studied. ${ }^{11,12}$ As seen previously, while some loop sequences hinder enzyme activity in our FRET-based assay, others improve target substrate cleavage, which is the presumed rate-limiting step of the sortase-catalyzed transpeptidation reaction. ${ }^{42}$ Here, we also use X-ray crystallography to look at the stereochemistry of both spySrtA and sagSrtA $\beta 7-\beta 8$ chimeric proteins. Finally, we use mutagenesis, structural, and sequence analyses to investigate conserved characteristics in the $\beta 7-\beta 8$ loops from Streptococcus SrtA proteins. Taken together, these analyses provide new insights on the role of conserved loops near the active site of Streptococcus Class A sortases. 
Gao and Johnson et al.

\section{Results}

Enzyme assays of wild-type S. pyogenes and S. agalactiae SrtA proteins

Based on our results using spSrtA, we designed a number of $\beta 7-\beta 8$ loop chimeras using spySrtA and sagSrtA as the "scaffolds." The wild-type sequences used were spySrtA82-249 (PDB ID 3FN5), sagSrtA79-238 (the sequence crystallized previously, in PDB ID 3RCC), or sagSrtA79-247, which includes the final nine C-terminal residues of sag SrtA based on UniProt ID SRTA_STRA3. ${ }^{43-45}$ For simplicity, we will refer to these as: spySrtA, sagSrtA 238 , and sagSrtA247. The $\beta 7-\beta 8$ loop sequences of these wild-type constructs were as follows: spySrtA (sequence: CTDIEATER, the catalytic Cys and Arg are included and underlined as reference points for the loop boundaries) and sagSrtA (ㄷDPEATER $)$. Notably, the $\beta 7-\beta 8$ loops of spySrtA and sagSrtA differ at only one position, 3 residues C-terminal to the catalytic Cys, which we will refer to as $\beta 7-\beta 8^{+3}$. The $\beta 7-\beta 8^{+3}$ residue is Ile in spySrtA and Pro in sagSrtA. The wild-type sequences of spySrtA and sagSrtA 247 are overall 65\% identical (Figure 2a), which is consistent with relative sequence identities amongst other representative Streptococcus Class A sortases (Figure S1).

In order to assess relative activity and selectivity, we used a FRET-based enzyme assay involving synthetic peptide substrates. This assay utilizes well-established FRET quencher probes consisting of a substrate sequence with an N-terminal 2-aminobenzoyl fluorophore (Abz) and C-terminal 2,4-dinitrophenyl (Dnp) quencher. ${ }^{12,14,46,47}$ For all assays, fluorescence was monitored for $2 \mathrm{~h}$ at room temperature and analyzed relative to a benchmark reaction consisting of wild-type saSrtA and the Abz-LPATGG-K(Dnp) peptide. ${ }^{12}$ For simplicity, we will remove the "Abz-" and "G-K(Dnp)" from peptide names hereafter, as they are not a part of the CWSS (e.g., AbzLPATGG-K(Dnp) will be referred to as LPATG). Additional experimental details are provided in the Materials and Methods and all averaged assay data and standard deviation values are in Table 
Gao and Johnson et al.

S1. All sortase enzymes were expressed and purified as previously described and as in the Materials and Methods. ${ }^{12}$ Purity was assessed by SDS-PAGE and monomeric protein fractions were pooled following size exclusion chromatography, as previously described. ${ }^{12}$

With the necessary materials in hand, we first evaluated the reactivity of wild-type spySrtA, sagSrtA 238 , and sagSrtA 247 proteins with peptides differing only at the P1' position (indicated in bold): LPATA, LPATG, and LPATS (Figure 2b). As our data shows, spySrtA was quite active, and exhibited robust reactivity that was comparable to the benchmark saSrtA/LPATG reaction. This protein also exhibited comparable reactivity at the $2 \mathrm{~h}$ reaction endpoint with G-, S-, and Acontaining peptides (Figure 2b). Activity for spySrtA was also markedly higher than spSrtA, which is consistent with a loop interaction described in our previous work. ${ }^{12}$ Specifically, the $\beta 6^{-2}$ position in spSrtA is R184, which was found to have a negative impact on reactivity that was attributed to a putative interaction with the $\beta 7-\beta 8^{-1}$ Glu of this enzyme. ${ }^{12}$ In spySrtA, the corresponding $\beta 6^{-2}$ position is $\mathrm{T} 185$, which likely minimizes this interaction and increases reactivity. Indeed, the spySrtA structure does not show evidence for this type of interaction (Figure S2a). Turning to the $S$. agalactiae constructs, sagSrtA 238 was catalytically inactive for all peptides tested, while sagSrtA247 reacted with all three, albeit at a lower level than spySrtA (Figure 2b). SagSrtA 247 also exhibited a preference for LPATA, and we observed $50 \%$ and $72 \%$ reductions in the relative activities for the G- and S-containing peptides as compared to LPATA, respectively. This is in contrast to spSrtA, which displayed almost identical relative fluorescence values after $2 \mathrm{~h}$ for G-, S-, and A-containing peptides $(0.29 \pm 0.04,0.26 \pm 0.02$, and $0.26 \pm 0.01$, respectively) (Figure 2b). ${ }^{12}$ 
Gao and Johnson et al.

Based on our previous results with spSrtA, we next wanted to substitute $\beta 7-\beta 8$ loop sequences from other SrtA proteins into spySrtA and sagSrtA $247 .{ }^{12}$ We chose to substitute the SrtA

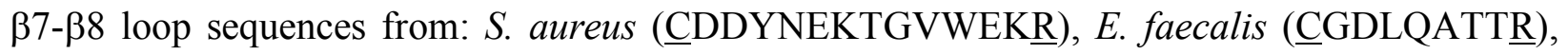

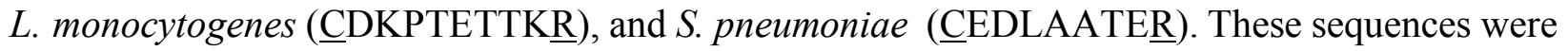
chosen due to their variable effects on spSrtA. ${ }^{12}$ For example, $\operatorname{spSrtA}_{\text {aureus }}$ (subscript denotes origin of the $\beta 7-\beta 8$ loop sequence) was relatively active and selective for a P1' Gly residue, spSrt $_{\text {faecalis }}$ was relatively active and non-selective at $\mathrm{P} 1$ ', and $\mathrm{spSrtA}_{\text {monocytogenes }}$ was inactive. $^{12}$

In total we tested eight additional variants: spySrt $\mathrm{A}_{\text {aureus, }}, \mathrm{spySrt}_{\mathrm{faecalis}}$, spySrt $\mathrm{A}_{\text {monocytogenes, }}$ spySrt $\mathrm{A}_{\text {pneumoniae, }}$ sagSrt $\mathrm{A}_{\text {aureus, }}, \quad$ sagSrt $\mathrm{A}_{\text {faecalis, }}$ sagSrt $\mathrm{A}_{\text {monocytogenes, }}$ and sagSrt $\mathrm{A}_{\text {pneumoniae. All }}$ proteins were expressed, purified and characterized as described previously and in the Materials and Methods. ${ }^{12}$ In general, we saw similar trends to those seen with spSrtA (Figure 3). ${ }^{12}$ For example, in the case of the $S$. aureus loop swaps we observed that both spySrtA aureus and sagSrtA $_{\text {aureus }}$ were selective for LPATG, as seen with spSrtA (Figure 3). All constructs containing the L. monocytogenes also showed a preference for LPATG, along with a clear reduction (2-3 fold) in activity as compared to the wild-type enzyme.

For the E. faecalis loop swaps, both the sagSrtA $\mathrm{Aaecalis}_{\text {and }}$ andSrt $\mathrm{A}_{\text {faecalis }}$ variants exhibited good reactivity that was generally higher than the corresponding wild-type enzyme. In contrast to spSrtA, however, the observed reactivity changes were not uniform across G-, S-, and Acontaining peptides. Specifically, for sagSrtA $_{\text {faecalis }}$ the relative activities for the LPATG and LPATS peptides were increased $\sim 2.5$-fold and 2.6-fold, respectively, as compared to wild-type, whereas for LPATA, it was only increased 1.2-fold (Figure 3b). In the case of spySrt $A_{\text {faecalis, }}$ analysis at the $2 \mathrm{~h}$ reaction timepoint initially suggested activity comparable to the wild-type enzyme and no preference for G-, S-, and A-containing peptides (Figure 3a). However, 
Gao and Johnson et al.

differences between spySrtA and spySrtA faecalis were evident at earlier reaction time points (Figure S3). In particular, at early stages in the reaction (e.g. $10 \mathrm{~min}$ ) the E. faecalis $\beta 7-\beta 8$ loop in spySrtA faecalis appeared to significantly increase reactivity with the G- and S-containing peptides ( $>2$-fold relative to spySrtA), and may have also had an effect on LPATA, but it is inconclusive due to large error bars for early time points in this reaction (Figure S3d).

Finally, installation of the $S$. pneumoniae loop resulted in decreases in activity for most enzyme-substrate combinations. For spySrtA pneumoniae, activity was $\sim 20 \%$ lower than spySrtA for all peptides tested. In sagSrt $\mathrm{A}_{\text {pneumoniae, }}$ the activity of the protein for the $\mathrm{G}$ - and $\mathrm{S}$-containing peptides was similar to sagSrtA 247 , but was reduced $~ 2.6$-fold for LPATA (Figure 3b). Focusing on spSrtA and spySrtA, comparison of the $\beta 7-\beta 8$ loop sequences revealed that while the final three positions of the 7-residue loop are identical (ATE), there are three differences in the first four positions (EDLA for spSrtA versus TDIE for spySrtA, differences in bold). Based on our previous work, we attribute the lower relative activities for the G-, S-, and A-containing peptides in spySrtA $_{\text {pneumoniae }}$ to the $\beta 7-\beta 8^{+1}$ Glu. ${ }^{12}$ Specifically, we predicted this may be due to an interaction with the $\beta 6^{-2} \mathrm{R} 184$ residue in spSrtA, which was supported by the observation that E208A and E208G, both at the $\beta 7-\beta 8^{+1}$ position, spSrtA mutants each revealed $\geq 2$-fold increases in relative reaction rates for all three peptides. ${ }^{12}$ Therefore, we in silico created T209E in the wild-type spySrtA structure to probe this hypothesis, and indeed saw that different rotamers of the mutated Glu are within distances consistent with forming a non-covalent interaction with the $\beta 6^{-2} \mathrm{~T} 185$ from the spySrtA scaffold (Figure S2b). Taken together, we consider this interaction to be a likely cause for the reduced activity in spySrtA pneumoniae.

Structure determination and analysis of wild-type sagSrtA 247 protein 
Gao and Johnson et al.

Our rationale for choosing to investigate the role of $\beta 7-\beta 8$ loop residues in $S$. pyogenes and S. agalactiae was to develop a structural model for probing these selectivity determinants. Both spySrtA and sagSrtA 238 were previously crystallized and we reasoned that experimental structural data would enable an understanding of the stereochemistry of sortase-substrate interactions in a way not available to spSrtA, which crystallizes as a catalytically inactive domain-swapped dimer. ${ }^{40,41,43,45}$

Beginning with sagSrtA, an important consideration for the reported sagSrtA 238 structure was that we found this variant to be inactive in our enzyme assays (Figure 2b). The crystal structure of sagSrtA 238 shows a dodecameric protein comprised of two hexameric rings (Figure S4a). The asymmetric unit contains one-and-a-half of these units, or 18 protomers total, with the other half of the second dodecamer present in a molecule related by symmetry. Each protomer is bound to three zinc ions, with additional ions modeled in the overall structure. There is no known biological requirement for higher order oligomers in sagSrtA activity or for zinc-binding and presumably, the presence of these ions is due to the crystallization conditions, e.g., zinc acetate or zinc sulfate. ${ }^{44}$ This previous work was focused on comparing the structures of sagSrt $\mathrm{A}_{238}$ with the S. agalactiae Class $\mathrm{C} 1$ sortase and did not include enzyme activity data. ${ }^{45}$ Of particular interest to us, however, is that in all 18 protomers of the sagSrtA 238 asymmetric unit, there are unresolved residues in either the $\beta 4-\beta 5$ loop, $\beta 7-\beta 8$ loop, or both (Figure S4b). Based on our enzyme assay data and previous structural analyses, we therefore sought to crystallize and determine a structure of sagSrtA 247 that would display relevant loop residues in an enzymatically active protein construct. $^{12}$

Crystallization conditions for sagSrtA 247 were identified using the Hampton PEGRx screen and optimized, as described in the Materials and Methods. We ultimately solved the structure of 
Gao and Johnson et al.

$\operatorname{sagSrtA}_{247}$ to $R_{\text {work }} / R_{\text {free }}=0.186 / 0.207$ at $1.4 \AA$ resolution, which includes residues Q82-L247

(Figure 4). All diffraction and refinement statistics are in Table 1.

The sagSrtA 247 structure adopts the conserved sortase fold, with a closed 8-stranded antiparallel $\beta$-sheet at its core (Figure 4a). ${ }^{2}$ Residues G225-F238, which are present in the crystallized sagSrtA 238 construct, but are not resolved in that crystal structure, form a C-terminal helix that directly interacts with residues in the $\beta 1, \beta 2, \beta 5$, and $\beta 6$ strands, in a hydrophobic manner (Figure 4b). There are also several hydrogen bonds formed in residues C-terminal to F238, specifically S239, K240, N243, and Q244 which are not present in the crystallized sagSrtA238 construct. These interactions are largely mediated by mainchain atoms, but also include the sidechains of S87, N104, K240, N243, and Q244 (Figure 4c). In addition, the side chain of I245 is a part of a hydrophobic pocket formed with V142, L145, and L152, which are residues in the $\beta 4-\beta 5$ loop (Figure 4d). We predict that the lack of these interactions destabilizes the sagSrtA 238 monomer, resulting in an inactive enzyme.

Interestingly, the C-terminus of saSrtA is substantially shorter than that of sagSrtA, or other Streptococcus SrtA proteins. Alignment of available saSrtA structures, including PDB IDs 1IJA (NMR), 1T2P (X-ray crystallography), and 2KID (NMR, +LPAT* peptidomimetic) indicate that the C-terminus of saSrtA, K206 (using 2KID and 1T2P numbering), corresponds stereochemically to K223 in sagSrtA (Figure S5a). ${ }^{15,20,48}$ Structural analyses of the two hydrophobic pockets that involve C-terminal residues in sagSrtA 247 suggest that the saSrtA sequence would be unlikely to accommodate a similar C-terminal extension (Figures S5b-c). Specifically, an overlay of relevant structures suggests steric clashes between E77 in saSrtA with F238 in sagSrtA 247 and R124 in saSrtA with I245 in sagSrtA 247 (Figures S5b-c). 
Gao and Johnson et al.

Structural alignments with protomers in sagSrtA238 (PDB ID 3RCC), spySrtA (3FN5), and S. mutans SrtA (4TQX) reveal that overall, sagSrtA247 adopts a conformation most similar to that of spySrtA (Figure S6a). Alignment with main chain atoms in each of the 18 protomers of the sagSrtA 238 asymmetric unit reveal an average root-mean squared deviation (RMSD) value of $0.690 \AA$ over 384 atoms, with the highest similarity between our structure and chain $\mathrm{O}(0.544 \AA$ over 371 atoms $)$ and lowest with chain K (0.792 $\AA$ over 394 atoms). Alignment with the two protomers of spySrtA revealed RMSD values of: $0.503 \AA$ (551 atoms, with chain A) and $0.475 \AA$ (535 atoms, chain B), and with $S$. mutans SrtA, the main-chain atoms align with an RMSD value of $0.566 \AA$ over 549 atoms (Figure S6a). The largest differences between sagSrtA 247 and spySrtA occur at the N-termini of both proteins (Figure S6b). In addition, we see $\sim 1 \AA$ shifts in two of the structurally-conserved loops that surround the peptide-binding cleft, the $\beta 4-\beta 5$ and $\beta 7-\beta 8$ loops, likely due to differences in crystal packing (Figure S6c). Notably, the side-chain location and orientation of residues in these loops previously identified as being selectivity determinants of spSrtA activity are stereochemically conserved (Figure S6d). ${ }^{12}$ Taken together, our structure suggests that the active sagSrtA protein adopts a similar monomeric conformation as spySrtA.

\section{Structural analyses of chimeric Streptococcus SrtA proteins}

We next wanted to investigate how our $\beta 7-\beta 8$ loop chimeras affect the structures of sagSrtA and spySrtA. We attempted to crystallize all 8 of our loop chimeras, using previously optimized conditions for sagSrtA and spySrtA, as well as by setting up commercially available crystal screens (e.g., Hampton PEG/ION, Index, and/or PEGRx). We were able to crystallize and solve structures of two of our chimeric proteins: spySrt $\mathrm{A}_{\text {faecalis }}$ and $\operatorname{spySrtA}_{\text {monocytogenes }}$ (Table 1). 
Gao and Johnson et al.

In addition, we successfully crystallized spySrt $\mathrm{A}_{\text {pneumoniae }}$ and sagSrt $\mathrm{A}_{\text {pneumoniae, }}$ but they were not of diffraction quality.

We resolved all residues of the $\beta 7-\beta 8$ loop of our spySrtA $A_{\text {faecalis }}$ structure (Figure S7a), and all but the middle two residues of the spySrtA monocytogenes loop (Figure S7b). The overall spySrtA variant conformations are identical to the wild-type protein, and alignments of mainchain atoms revealed RMSD values of $0.351 \AA$ (533 atoms) and $0.252 \AA$ (488 atoms) for spySrt $\mathrm{A}_{\text {faecalis }}$ and spySrtA $_{\text {monocytogenes, }}$ respectively (Figure S7c).

In the spySrtA variant structures, the orientation of the spySrt $\mathrm{A}_{\text {faecalis }}$ loop is very similar to the wild-type protein (Figure 5a), and the intra-loop hydrogen bond between the conserved $\beta 7-\beta 8^{+2}$ Asp and $\beta 7-\beta 8^{+6} \mathrm{Thr}$ is maintained. This is not the case in the spySrtA $\mathrm{A}_{\text {monocytogenes }} \beta 7-\beta 8$ loop, as compared to the L. monocytogenes SrtA (lmSrtA) structure (Figures 5b-c) ${ }^{49}$ Here, the wild-type position of the $\beta 7-\beta 8^{+3}$ Pro sterically clashes with the $\beta 4-\beta 5^{+3}$ F145 residue in spySrtA and as a result, the $\beta 7-\beta 8^{+3}$ Pro in spySrt $\mathrm{A}_{\text {monocytogenes }}$ is shifted away relative to the $\beta 4-\beta 5$ loop (Figures 5b-c). This results in breakage of the intra-loop hydrogen bond; whereas the distance between $\beta 7-\beta 8^{+1} \mathrm{D} 118$ and $\beta 7-\beta 8^{+6} \mathrm{~T} 123$ is $2.8 \AA$ in $\operatorname{lmSrtA}$, it is $7.7 \AA$ in spySrt $\mathrm{A}_{\text {monocytogenes }}$ (black arrows in Figures 5b-c).

In the active conformation of saSrtA (PDB ID 2KID), the 12 residues in the $\beta 7-\beta 8$ loop adopt a tight structure, mediated by several intraloop hydrogen bonds and noncovalent interactions, all of which include the sidechain atoms of N188 (Figures 5e, S7d). Therefore, we wanted to test the contribution of this residue on the spySrtA aureus and sagSrtA aureus proteins, with the variants $\Delta \mathrm{N} 188$ spySrtA $\mathrm{A}_{\text {aureus }}$ and $\Delta \mathrm{N} 188$ sagSrtA $_{\text {aureus }}$ (Figures 3a-b). These proteins were similar to other saSrtA loop variants in that they were selective for LPATG; however, the relative activities were reduced by half as compared to spySrtA aureus and 4.5-fold as compared to 
Gao and Johnson et al.

sagSrtA aureus, respectively (Figures 3a-b). We next crystallized and solved the structure of $\Delta \mathrm{N} 188$

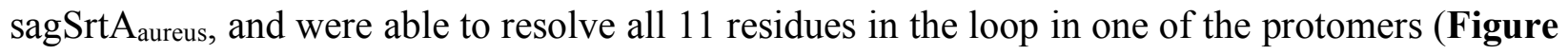
S7e). Alignment of the sagSrtA 247 and $\Delta \mathrm{N} 188$ sagSrtA $_{\text {aureus }}$ structures reveals the greatest structural variability in the $\beta 6-\beta 7$ and $\beta 7-\beta 8$ loops, and the overall $\mathrm{RMSD}=0.298 \AA$ (496 mainchain atoms) (Figure S7f).

Comparison of the $\beta 7-\beta 8$ loops in $\Delta \mathrm{N} 188$ sagSrtA $_{\text {aureus }}$ and sagSrtA 247 indicate that the loop in $\Delta \mathrm{N} 188$ sagSrt $\mathrm{A}_{\text {aureus }}$ adopts a more open shape (Figure 5d). In the absence of N188, it is unsurprising that the $\beta 7-\beta 8$ loop in $\Delta \mathrm{N} 188$ sagSrt $\mathrm{A}_{\text {aureus }}$ is missing the equivalent saSrtA interactions and shows only very weak, and likely unfavorable repulsive electrostatic ones between D185 and E195 (Figure S7g). Finally, we see displacement of the W194 residue in the $\Delta$ N188 sagSrtA $A_{\text {aureus }}$ structure (Figure 5f). We hypothesize this is the largest contributor to the weaker activity in $\Delta \mathrm{N} 188$ sagSrtA $_{\text {aureus, }}$ as mutation to alanine at this residue was previously shown to reduce the activity of saSrtA by approximately 2-fold, which is of a similar magnitude to the effect of $\Delta \mathrm{N} 188$ on spySrtA aureus $\left(\right.$ Figure 3a-b). ${ }^{14}$ Interestingly, despite differences in overall shape of the $\beta 7-\beta 8$ loop in $\Delta \mathrm{N} 188$ sagSrtA $\mathrm{A}_{\text {aureus, }}$ the protein retains the stringent selectivity of the saSrtA protein, recognizing only LPATG (Figure 3b).

\section{Mutagenesis of Streptococcus SrtA proteins}

Based on our structures, it is not immediately clear why sagSrtA 247 is less active than spySrtA (Figures 3a-b). It is also not obvious why, for example, the $\Delta$ N188 mutation described above reduced spySrtA $\mathrm{A}_{\text {aureus }}$ activity 2-fold, but sagSrtA aureus activity 4.5-fold, or why there appears to be reduced ability to recognize S-containing peptides for several of the sagSrtA variants, as compared to spySrtA (Figures 3a-b). In the vicinity of the peptide-binding cleft, there are 4 
Gao and Johnson et al.

non-conservative mutations (Figure 6a). Of these, we identified two that may contribute directly to the relatively low activity of sagSrtA247: K183 and P209.

Beginning with the $\mathrm{K} 183$ residue of sagSrtA 247 , we noted that it occupied the $\beta 6^{-2}$ position of the enzyme. In spySrtA, a threonine (T185) is present at the $\beta 6^{-2}$ position, which should not interact with the $\beta 7-\beta 8^{-1} \mathrm{Glu}$, thereby avoiding an interaction that was previously shown to reduce enzyme activity in spSrtA. ${ }^{12}$ The Lys substitution in sagSrtA, however, would allow the $\beta 6^{-2} \mathrm{~K} 183$ to interact with the $\beta 7-\beta 8^{-1}$ E213 (Fig $\mathbf{6 b}$ ), and potentially reduce activity in a manner similar to the hypothesized interaction of the $\beta 6^{-2}$ R184 with $\beta 7-\beta 8^{-1}$ E214 in spSrtA. ${ }^{12}$

With respect to the second residue (P209), we noticed that it occupied the $\beta 7-\beta 8^{+3}$ loop position in sagSrtA 247 , similar to that in 1 SrtA. We previously hypothesized that the $\beta 7-\beta 8^{+3}$ Pro negatively affected spSrtA $\mathrm{A}_{\text {monoytogenes }}$ and mutation of the wild-type Leu in L209P spSrtA reduced activity by about 2 -fold. ${ }^{12}$ To test this, we mutated the $\beta 7-\beta 8^{+3}$ loop residue in sagSrt $\mathrm{A}_{247}$ and spySrtA to that of the other protein, or P209I sagSrtA 247 and I211P spySrtA, respectively. Relative enzyme activities were assayed and indeed, we saw an 2-fold increase in activity for P209I sagSrtA for G-, S-, and A-containing peptides (Figure 6c). Interestingly, we saw minimal differences in the activities of I211P spySrtA as compared to wild-type spySrtA, suggesting that the $\beta 7-\beta 8^{+3}$ residue interaction may not be as critical for spySrtA, which is relatively more active than either sagSrtA or spSrtA (Figures 2b).

Sequence Patterns in the $\beta 7$ - $\beta 8$ loops of Streptococcus SrtA proteins

Finally, we wanted to gain a general understanding of sequence patterns in the $\beta 7-\beta 8$ loops of Streptococcus SrtA proteins. Therefore, we created a WebLogo of $37 \quad \beta 7-\beta 8$ loops from Streptococcus SrtA proteins from the UniProt database (Figure 6d, Table S2). ${ }^{50,51}$ We identified 
Gao and Johnson et al.

the loop sequences by using the catalytic cysteine and arginine residues to mark the N- and Cterminal residues of the $\beta 7-\beta 8$ loop, respectively. Our WebLogo analysis agreed with our biochemical and structural observations. The $\beta 7-\beta 8^{+2}$ residue is an Asp in all of the loops, while the $\beta 7-\beta 8^{+6}$ residue is a Thr (or Ser) in $35 / 37$ sequences, consistent with an intraloop hydrogen bond interaction observed here and previously. ${ }^{12}$ We also observed an interaction between the $\beta 7-\beta 8^{+3}$ position and the $\beta 4-\beta 5$ loop, typically of a hydrophobic nature. ${ }^{12}$ Consistent with this, our WebLogo analysis showed that the $\beta 7-\beta 8^{+3}$ position is hydrophobic (Ala, Ile, Leu, Met, or Tyr) in 31 of the sequences, with the remaining sequences containing either Gln (5/37 sequences) or a Pro that is present in only the sagSrtA enzyme. It is unclear if the Gln can interact with the $\beta 4-\beta 5$ residues previously identified and if, as discussed for spySrtA, this interaction is correlated to the presence of a $\beta 7-\beta 8^{-1} / \beta 6^{-2}$ interaction.

Notably, all 37 sequences contain a $\beta 7-\beta 8^{+5}$ Ala residue (Table S2). Analyses of this residue in the sagSrtA 247 and spySrtA structures show that it is solvent exposed (Figure S8). However, alignment of sagSrtA 247 with saSrtA-LPAT* (2KID) revealed that the $\beta 7-\beta 8^{+5}$ A211 sidechain points directly towards the peptide (Figure 6e). Furthermore, the carbonyl of A211 interacts with the guanidinium group of the catalytic arginine and the Ala is in the same stereochemical position as W194 in saSrtA (Figure 6e). Taken together, this suggests that the $\beta 7-\beta 8^{+5}$ Ala residue in Streptococcus SrtA proteins may play an important role in enzyme function, just as the $\beta 7-\beta 8^{+10}$ Trp residue does in saSrtA. 
Gao and Johnson et al.

\section{Discussion}

Work from ourselves and others indicates that the structurally conserved, yet sequence variable, $\beta 6-\beta 7$ and $\beta 7-\beta 8$ loops in Class A sortases directly affect target recognition and enzyme activity. ${ }^{12,38,39}$ Previously, we used the Class A sortase from Streptococcus pneumoniae to investigate the differences in selectivity and activity at the P1' position as compared to SrtA from Staphylococcus aureus and 7 other organisms. ${ }^{11,12}$ Here, we extended these studies to look at similar chimeric SrtA enzymes from Streptococcus pyogenes and Streptococcus agalactiae, which were previously crystallized. ${ }^{43-45}$ Using protein biochemistry and structural biology, we find additional evidence in support of our hypothesis that the $\beta 7-\beta 8$ loop residues in these proteins determine overall enzyme activity and selectivity in a similar manner to spSrtA. Specifically, our data strongly supports the presence of 3 interactions mediated by $\beta 7-\beta 8$ loop residues in Streptococcus SrtA proteins that can mediate enzyme function. ${ }^{12}$ Although the exact nature of these interactions can vary in SrtA proteins from different organisms, e.g., Staphylococcus aureus, we argue that related ones are likely present across the broad sortase superfamily.

Our work also highlights the need for additional sortase structures that are paired with biochemical data. For example, we discovered that the sagSrtA construct previously crystallized is of an inactive enzyme, and omits several important C-terminal interactions. ${ }^{44,45}$ This is also notable because the C-terminus of saSrtA is substantially shorter than that of the Streptococcus SrtA proteins and without the biochemical knowledge of enzyme activity, fundamental information about these enzymes is missed. In addition, the only available spSrtA structures in the Protein Data Bank are of domain-swapped dimers, which are not enzymatically active in our hands (data not shown). ${ }^{40,41}$ Considering the observations about contributions of individual residues to 
Gao and Johnson et al.

activity and/or selectivity by ourselves and others, there remains much to be learned from the study of individual sortase enzymes.

Finally, the work presented here may have implications for the continued development of sortase mediated-ligation as a tool for protein engineering. Recent applications of sortagging in cells and the evolution of sortases to recognize specific targets for potential therapeutics are amongst a number of exciting developments in the field. ${ }^{6,52}$ A greater understanding of substrate selectivity and target recognition could enable more sophisticated orthogonal labeling schemes in which multiple sortase enzymes can be utilized to recognize and modify distinct sequences on a single protein or simultaneous labeling of multiple targets. ${ }^{3,9,53,54}$ This ability to add numerous site-specific tags to protein targets in vitro and in vivo would be a powerful addition to the arsenal of protein engineering. 
Gao and Johnson et al.

\section{Experimental Procedures}

Sequences used. The wild-type spySrtA sequence used is from the published structure, PDB ID 3FN5. This sequence was originally amplified from serotype M1 S. pyogenes strain SF370 genomic DNA, as previously described. ${ }^{43}$ This sequence is $74 \%$ identical ( $85 \%$ similar) to the S. pyogenes Class A sortase in UniProt, A0A2W5CEK0_STRPY (unreviewed). The wild-type sagSrtA sequence used is from the published structure, PDB ID 3RCC. This sequence was originally amplified from genomic DNA of $S$. agalactiae strain 2603V/R (locus tag of SAG0961), as previously described. ${ }^{44}$ This sequence is $99 \%$ identical, differing only at Q132, which is a proline in 3RCC, to $S$. agalactiae SrtA in UniProt, SRTA_STRA3 (reviewed). This substitution occurs in the $\beta 3-\beta 4$ loop. All constructs in this work, including chimeric and mutant proteins, were purchased from Genscript in the $\mathrm{pET} 28 \mathrm{a}(+)$ vector.

Protein expression and purification. All proteins were expressed and purified as previously described for related SrtA proteins. ${ }^{11,12}$ Briefly, plasmids were transformed into Escherichia coli BL21 (DE3) competent cells and grown in LB media, with protein induction at $\mathrm{OD}_{600}$ 0.6-0.8 using $0.15 \mathrm{mM}$ IPTG for $18-20 \mathrm{~h}$ at $18^{\circ} \mathrm{C}$. The cells were harvested in lysis buffer [0.05 M Tris pH 7.5, $0.15 \mathrm{M} \mathrm{NaCl}, 0.5 \mathrm{mM}$ ethylenediaminetetraacetic acid (EDTA)] and whole cell lysate was clarified using centrifugation. The supernatant was filtered and loaded onto a $5 \mathrm{~mL}$ HisTrap HP column (GE Life Sciences, now Cytiva), followed by washing [0.05 M Tris pH 7.5, 0.15 M NaCl, 0.02 M imidazole, 0.001 M TCEP] and then elution [wash buffer with $0.3 \mathrm{M}$ imidazole] of the desired protein. The His-tags of proteins prepared for crystallography were proteolyzed using Tobacco Etch Virus (TEV) protease overnight at $4{ }^{\circ} \mathrm{C}$ and a ratio of $\sim 1: 100$ (TEV:protein). The His6-TEV sequence was left on proteins used for enzyme assays. Size exclusion chromatography (SEC) was 
Gao and Johnson et al.

conducted using a HiLoad 16/600 Superdex 75 column (GE Life Sciences, now Cytiva) in SEC running buffer [0.05 $\mathrm{M}$ Tris $\mathrm{pH}$ 7.5, 0.15 M NaCl, 0.001 M TCEP]. Purified protein corresponding to the monomeric peak was concentrated using an Amicon Ultra-15 Centrifugal Filter Unit (10,000 NWML) and analyzed by SDS-PAGE and analytical SEC. ${ }^{12}$ Protein concentrations were determined using theoretical extinction coefficients calculated using ExPASy ProtParam. ${ }^{55}$ Protein not immediately used was flash frozen in SEC running buffer and stored at $-80^{\circ} \mathrm{C}$.

Crystallization. Prior to crystallization, spySrtA variants were dialyzed into crystallization buffer [20 mM Tris $\mathrm{pH} 7.5,150 \mathrm{mM} \mathrm{NaCl}]$, based on previously published conditions. ${ }^{43}$ The protein concentrations used for crystallization were as follows: sagSrtA $247(15 \mathrm{mg} / \mathrm{mL}), \Delta \mathrm{N} 188$ $\operatorname{sagSrtA}_{\text {aureus }}(16 \mathrm{mg} / \mathrm{mL}), \operatorname{spySrtA}_{\text {faecalis }}(42 \mathrm{mg} / \mathrm{mL})$, and spySrtA $\mathrm{A}_{\text {monocytogenes }}(20 \mathrm{mg} / \mathrm{mL})$. The proteins were crystallized using the hanging drop vapor diffusion technique with well and protein solution mixed in a 1:1 ratio $(2 \mu \mathrm{l}: 2 \mu \mathrm{l})$. Crystallization conditions for the spySrtA variants were optimized using the crystal conditions for the apo protein. ${ }^{43}$ For sagSrtA 247 , initial crystallization conditions were identified using the PEGRx screen from Hampton Research. The crystallization conditions of the crystals used for data collection were: sagSrtA247 [20\% $(v / v)$ 2-Propanol, $0.1 \mathrm{M}$ MES monohydrate pH 6.1, 20\% (w/v) PEG monomethyl ether 2,000], $\Delta$ N188 sagSrtA aureus $[12 \%$ $(v / v)$ 2-Propanol, 0.02 M MES monohydrate $\mathrm{pH} 6,24 \%(w / v)$ PEG monomethyl ether 2,000], spySrtA faecalis $[0.2 \mathrm{M}$ sodium acetate, $0.1 \mathrm{M}$ Tris $\mathrm{pH}$ 6, 30\% (w/v) PEG 8,000], and spySrtA $_{\text {monocytogenes }}[0.2 \mathrm{M}$ sodium acetate, $0.1 \mathrm{M}$ Tris $\mathrm{pH} 6.5,24 \%(w / v)$ PEG 8,000]. For all proteins, glycerol was used as a cryoprotectant and the cryo solutions were equal to crystallization conditions plus $20 \%(v / v)$ glycerol for all except sagSrtA 247 (plus $15 \%(v / v)$ glycerol). The crystals were flash-cooled by plunging into liquid nitrogen. 
Gao and Johnson et al.

Data collection, structure determination, and protein analyses. Initial data for sagSrtA 247 were collected to $2.0 \AA \AA$ on a Bruker Apex CCD diffractometer at $\lambda=1.54056 \mathrm{~nm}$. Data were collected at the Advanced Light Source (ALS) at Lawrence Berkeley National Laboratory (LBNL) on beamline 5.0.1 and 5.0.2, at $\lambda=1.00004 \mathrm{~nm}$ or $0.99988 \mathrm{~nm}$ over $360^{\circ}$, with $\Delta \phi=0.25^{\circ}$ frames and an exposure time of $0.5 \mathrm{~s}$ per frame. Data were processed using the XDS package (Table 1). ${ }^{56,57}$ Molecular Replacement was performed using Phenix with the following search models: spySrtA (PDB ID 3FN5) for spySrtA faecalis and spySrtA monocytogenes, sagSrtA238 (3RCC) for sagSrtA 247 and sagSrtA $_{247}$ for $\Delta \mathrm{N} 188$ sagSrtA $_{\text {aureus. }}$ Refinement was performed using Phenix, manual refinement was done using Coot, and model geometry was assessed using MolProbity and the PDB validation server. ${ }^{58-60}$ Phenix.Xtriage was also used to assess data quality, specifically to identify a number of outliers in the spySrtA monocytogenes data. ${ }^{60}$ All crystal data and refinement statistics are in Table 1. Sequence alignments were performed using T-coffee or BlastP. ${ }^{61,62}$ Visualization of alignments were done using Jalview or Boxshade. ${ }^{63}$ WebLogo was also used to visualize sequences. ${ }^{64}$ Structural analyses and figure rendering were done using PyMOL. PDB accession codes for the structures presented here are: $\operatorname{sagSrtA}_{247}(7 \mathrm{~S} 56), \Delta \mathrm{N} 188$ sagSrtA $\mathrm{A}_{\text {aureus }}(7 \mathrm{~S} 54)$, spySrtA faecalis (7S57), and spySrtA monocytogenes (7S53).

Peptide synthesis. Model peptide substrates were synthesized via manual Fmoc solid phase peptide synthesis (SPPS) as previously described. ${ }^{12}$

Fluorescence Assay for Sortase Activity. Enzyme assays were conducted using a Biotek Synergy H1 plate reader as previously described. ${ }^{12}$ The fluorescence intensity of each well was measured 
bioRxiv preprint doi: https://doi.org/10.1101/2021.09.19.461001; this version posted September 19, 2021. The copyright holder for this preprint

(which was not certified by peer review) is the author/funder, who has granted bioRxiv a license to display the preprint in perpetuity. It is made available under aCC-BY-NC-ND 4.0 International license.

Gao and Johnson et al.

at 2-min time intervals over a 2 -hr period at room temperature $\left(\lambda_{\mathrm{ex}}=320 \mathrm{~nm}, \lambda_{\mathrm{em}}=420 \mathrm{~nm}\right.$, and detector gain $=75$ ). All reactions were performed in at least triplicate. For each substrate sequence, the background fluorescence of the intact peptide in the absence of enzyme was subtracted from the observed experimental data. Background-corrected fluorescence data was then normalized to the fluorescence intensity of a benchmark reaction between wild-type saSrtA and Abz-LPATGG-K(Dnp). ${ }^{12}$ Data figures were prepared using GraphPad Prism 9.1.2. 
Gao and Johnson et al.

\section{Acknowledgements}

The authors would like to thank the other members of the Amacher and Antos labs for helpful discussions and assistance. They would also like to thank the Berkeley Center for Structural Biology (BCSB) for being an excellent resource for the crystallography community. The BCSB is supported in part by the National Institutes of Health, National Institute of General Medical Sciences, and the Howard Hughes Medical Institute. The Advanced Light Source is supported by the Director, Office of Science, Office of Basic Energy Sciences, of the U.S. Department of Energy under Contract No. DE-AC02-05CH11231. Other grant information: JFA and JMA were both funded by Cottrell Scholar Awards from the Research Corporation for Science Advancement. JFA was also funded by NSF CHE-CAREER-2044958. The Rigaku X-ray Diffractometer was funded by NSF CHE-MRI-1429164 and used to collect initial sagSrtA 247 diffraction data. In addition, IMP and HMK received Elwha Undergraduate Summer Research Awards and DAJ received a Joseph \& Karen Morse Student Research in Chemistry Fellowship to fund summer research.

\section{Declaration of interests}

The authors declare no competing interest. 
Gao and Johnson et al.

Table 1. Data Collection and Refinement Statistics.

\begin{tabular}{|c|c|c|c|c|}
\hline & sagSrtA247 & $\begin{array}{c}\Delta \mathrm{N188} \\
\text { sagSrtA }_{\text {aureus }} \\
\end{array}$ & spySrt $A_{\text {faecalis }}$ & spySrtAmonocytogenes \\
\hline \multicolumn{5}{|l|}{ Data Collection } \\
\hline Space Group & $P 2{ }_{1} 22_{1}(19)$ & $I 2(5)$ & $P 2{ }_{1} 2_{1} 2_{1}(19)$ & $P 2{ }_{1} 2_{1} 2_{1}(19)$ \\
\hline \multicolumn{5}{|l|}{ Unit cell dimensions } \\
\hline$a, b, c(\AA)$ & $44.13,54.64,60.25$ & $71.30,33.52,115.6$ & $33.60,56.92,71.35$ & $34.29,58.14,73.27$ \\
\hline$\alpha, \beta, \gamma\left({ }^{\circ}\right)$ & $90,90,90$ & $90,91.8,90$ & $90,90,90$ & $90,90,90$ \\
\hline $\operatorname{Resolution}^{\mathrm{a}}(\AA)$ & $40.5-1.4(1.5-1.4)$ & $35.6-1.8(1.9-1.8)$ & $44.5-1.7(1.8-1.7)$ & $45.5-1.6(1.7-1.6)$ \\
\hline $\mathrm{R}_{s y m}^{\mathrm{b}}(\%)$ & $5.3(69.4)$ & $9.6(111.4)$ & $7.1(190.9)$ & $6.7(44.2)$ \\
\hline $\mathrm{I} / \sigma_{\mathrm{I}}$ & $29.08(3.78)$ & $11.60(1.53)$ & $22.91(1.5)$ & $16.36(3.02)$ \\
\hline Completeness $(\%)$ & $99.6(98.6)$ & $99.3(98.0)$ & $99.7(98.0)$ & $98.5(99.6)$ \\
\hline \multicolumn{5}{|l|}{ Refinement } \\
\hline Total \# of reflections & 29,340 & 25,788 & 15,645 & 19,635 \\
\hline $\begin{array}{l}\text { Reflections in the test } \\
\text { set }\end{array}$ & 1,463 & 1,254 & 769 & 960 \\
\hline$R_{\text {work }} / R_{\text {free }}{ }^{\mathrm{e}}$ & $18.5 / 20.7$ & $18.5 / 24.9$ & $18.0 / 21.7$ & $26.3 / 30.1$ \\
\hline \multicolumn{5}{|l|}{ Number of atoms: } \\
\hline Protein & 1277 & 2567 & 1245 & 1241 \\
\hline Water & 206 & 213 & 107 & 228 \\
\hline Ramachandran $\operatorname{plot}^{\mathrm{f}}(\%)$ & $99.38 / 0.62 / 0$ & $97.23 / 2.77 / 0$ & $99.37 / 0.63 / 0$ & $97.39 / 1.96 / 0.65$ \\
\hline \multicolumn{5}{|l|}{$B_{\text {av }}\left(\AA^{2}\right)$} \\
\hline Protein & 15.70 & 17.89 & 24.85 & 15.24 \\
\hline Bond length RMSD $(\AA)$ & 0.010 & 0.007 & 0.007 & 0.006 \\
\hline Bond angle RMSD $\left(^{\circ}\right)$ & 1.102 & 0.943 & 0.949 & 0.925 \\
\hline
\end{tabular}

${ }^{a}$ Values in parentheses are for data in the highest-resolution shell.

${ }^{\mathrm{b}} R_{\mathrm{sym}}=\Sigma_{h} \Sigma_{i}\left|\mathrm{I}(h)-\mathrm{I}_{i}(h)\right| / \Sigma_{h} \Sigma_{i} \mathrm{I}_{i}(h)$, where $\mathrm{I}_{i}(h)$ and $\mathrm{I}(h)$ values are the $i$-th and mean measurements of the intensity of reflection $h$.

${ }^{\mathrm{c}}$ SigAno $=|\mathrm{F}(+)-\mathrm{F}(-)| / \sigma$

${ }^{\mathrm{d}} R_{\text {work }}=\Sigma|| \mathrm{F}_{\text {obs }}|h-| \mathrm{F}_{\text {calc }} \|_{h} / \Sigma\left|\mathrm{F}_{\text {obs }}\right| h, h \in$ \{working set $\}$

${ }^{\mathrm{e}} R_{\text {free }}$ is calculated as $R_{\text {work }}$ for the reflections $h \in$ \{test set\}

${ }^{\mathrm{f}}$ Favored/allowed/outliers 
Gao and Johnson et al.

\section{References}

1. Spirig T, Weiner EM, Clubb RT (2011) Sortase enzymes in Gram-positive bacteria. Mol. Microbiol. 82:1044-1059.

2. Jacobitz AW, Kattke MD, Wereszczynski J, Clubb RT (2017) Sortase transpeptidases: structural biology and catalytic mechanism. Adv. Protein Chem. Struct. Biol. 109:223-264.

3. Antos JM, Truttmann MC, Ploegh HL (2016) Recent advances in sortase-catalyzed ligation methodology. Curr. Opin. Struct. Biol. 38:111-118.

4. Bradshaw WJ, Davies AH, Chambers CJ, Roberts AK, Shone CC, Acharya KR (2015) Molecular features of the sortase enzyme family. FEBS J. 282:2097-2114.

5. Dorr BM, Ham HO, An C, Chaikof EL, Liu DR (2014) Reprogramming the specificity of sortase enzymes. Proc. Natl. Acad. Sci. USA 111:13343-13348.

6. Podracky CJ, An C, DeSousa A, Dorr BM, Walsh DM, Liu DR (2021) Laboratory evolution of a sortase enzyme that modifies amyloid- $\beta$ protein. Nat. Chem. Biol. 17:317-325.

7. Chen I, Dorr BM, Liu DR (2011) A general strategy for the evolution of bond-forming enzymes using yeast display. Proc. Natl. Acad. Sci. USA 108:11399-11404.

8. Piotukh K, Geltinger B, Heinrich N, Gerth F, Beyermann M, Freund C, Schwarzer D (2011) Directed evolution of sortase A mutants with altered substrate selectivity profiles. J. Am. Chem. Soc. 133:17536-17539.

9. Antos JM, Chew G-L, Guimaraes CP, Yoder NC, Grotenbreg GM, Popp MW-L, Ploegh HL (2009) Site-specific N- and C-terminal labeling of a single polypeptide using sortases of different specificity. J. Am. Chem. Soc. 131:10800-10801.

10. Schmohl L, Bierlmeier J, Gerth F, Freund C, Schwarzer D (2017) Engineering sortase A by screening a second-generation library using phage display. J Pept Sci 23:631-635.

11. Nikghalb KD, Horvath NM, Prelesnik JL, Banks OGB, Filipov PA, Row RD, Roark TJ, Antos JM (2018) Expanding the Scope of Sortase-Mediated Ligations by Using Sortase Homologues. Chembiochem 19:185-195.

12. Piper IM, Struyvenberg SA, Valgardson JD, Alex Johnson D, Gao M, Johnston K, Svendsen JE, Kodama HM, Hvorecny KL, Antos JM, et al. (2021) Sequence variation in the $\beta 7-\beta 8$ loop of bacterial Class A sortase enzymes alters substrate selectivity. J. Bio. Chem.:100981.

13. Malik A, Kim SB (2019) A comprehensive in silico analysis of sortase superfamily. J. Microbiol. 57:431-443.

14. Ton-That H, Mazmanian SK, Alksne L, Schneewind O (2002) Anchoring of surface proteins to the cell wall of Staphylococcus aureus. Cysteine 184 and histidine 120 of sortase form a thiolateimidazolium ion pair for catalysis. J. Biol. Chem. 277:7447-7452.

15. Suree N, Liew CK, Villareal VA, Thieu W, Fadeev EA, Clemens JJ, Jung ME, Clubb RT (2009) The structure of the Staphylococcus aureus sortase-substrate complex reveals how the universally conserved LPXTG sorting signal is recognized. J. Biol. Chem. 284:24465-24477.

16. Naik MT, Suree N, Ilangovan U, Liew CK, Thieu W, Campbell DO, Clemens JJ, Jung ME, Clubb RT (2006) Staphylococcus aureus Sortase A transpeptidase. Calcium promotes sorting signal binding by altering the mobility and structure of an active site loop. J. Biol. Chem. 281:1817-1826.

17. Kappel K, Wereszczynski J, Clubb RT, McCammon JA (2012) The binding mechanism, multiple binding modes, and allosteric regulation of Staphylococcus aureus Sortase A probed by molecular dynamics simulations. Protein Sci. 21:1858-1871.

18. Ugur I, Schatte M, Marion A, Glaser M, Boenitz-Dulat M, Antes I (2018) Ca2+ binding 
Gao and Johnson et al.

induced sequential allosteric activation of sortase A: An example for ion-triggered conformational selection. PLoS One 13:e0205057.

19. Tee W-V, Guarnera E, Berezovsky IN (2020) Disorder driven allosteric control of protein activity. Current Research in Structural Biology 2:191-203.

20. Ilangovan U, Ton-That H, Iwahara J, Schneewind O, Clubb RT (2001) Structure of sortase, the transpeptidase that anchors proteins to the cell wall of Staphylococcus aureus. Proc. Natl. Acad. Sci. USA 98:6056-6061.

21. Chan AH, Yi SW, Terwilliger AL, Maresso AW, Jung ME, Clubb RT (2015) Structure of the Bacillus anthracis Sortase A Enzyme Bound to Its Sorting Signal: A FLEXIBLE AMINOTERMINAL APPENDAGE MODULATES SUBSTRATE ACCESS. J. Biol. Chem. 290:2546125474.

22. Goettig P, Brandstetter H, Magdolen V (2019) Surface loops of trypsin-like serine proteases as determinants of function. Biochimie 166:52-76.

23. Hedstrom L, Szilagyi L, Rutter WJ (1992) Converting trypsin to chymotrypsin: the role of surface loops. Science 255:1249-1253.

24. Pirola L, Zvelebil MJ, Bulgarelli-Leva G, Van Obberghen E, Waterfield MD, Wymann MP (2001) Activation loop sequences confer substrate specificity to phosphoinositide 3-kinase alpha (PI3Kalpha ). Functions of lipid kinase-deficient PI3Kalpha in signaling. J. Biol. Chem. 276:21544-21554.

25. Shah NH, Wang Q, Yan Q, Karandur D, Kadlecek TA, Fallahee IR, Russ WP, Ranganathan R, Weiss A, Kuriyan J (2016) An electrostatic selection mechanism controls sequential kinase signaling downstream of the T cell receptor. Elife 5:e20105.

26. Laham LE, Mukhopadhyay N, Roberts TM (2000) The activation loop in Lck regulates oncogenic potential by inhibiting basal kinase activity and restricting substrate specificity. Oncogene 19:3961-3970.

27. Kunz J, Wilson MP, Kisseleva M, Hurley JH, Majerus PW, Anderson RA (2000) The activation loop of phosphatidylinositol phosphate kinases determines signaling specificity. Mol. Cell 5:1-11.

28. Nolen B, Taylor S, Ghosh G (2004) Regulation of protein kinases; controlling activity through activation segment conformation. Mol. Cell 15:661-675.

29. Liu H, Huang H, Voss C, Kaneko T, Qin WT, Sidhu S, Li SS-C (2019) Surface loops in a single $\mathrm{SH} 2$ domain are capable of encoding the spectrum of specificity of the SH2 family. Mol. Cell Proteomics 18:372-382.

30. Kaneko T, Huang H, Zhao B, Li L, Liu H, Voss CK, Wu C, Schiller MR, Li SS-C (2010) Loops govern SH2 domain specificity by controlling access to binding pockets. Sci. Signal. 3:ra34. 31. Kazlauskas A, Schmotz C, Kesti T, Hepojoki J, Kleino I, Kaneko T, Li SSC, Saksela K (2016) Large-Scale Screening of Preferred Interactions of Human Src Homology-3 (SH3) Domains Using Native Target Proteins as Affinity Ligands. Mol. Cell Proteomics 15:3270-3281.

32. Songyang Z, Shoelson SE, McGlade J, Olivier P, Pawson T, Bustelo XR, Barbacid M, Sabe $\mathrm{H}$, Hanafusa H, Yi T (1994) Specific motifs recognized by the SH2 domains of Csk, 3BP2, fps/fes, GRB-2, HCP, SHC, Syk, and Vav. Mol. Cell. Biol. 14:2777-2785.

33. Teyra J, Huang H, Jain S, Guan X, Dong A, Liu Y, Tempel W, Min J, Tong Y, Kim PM, et al. (2017) Comprehensive Analysis of the Human SH3 Domain Family Reveals a Wide Variety of Non-canonical Specificities. Structure 25:1598-1610.e3.

34. Li SS-C (2005) Specificity and versatility of SH3 and other proline-recognition domains: structural basis and implications for cellular signal transduction. Biochem. J. 390:641-653. 
Gao and Johnson et al.

35. Sparks AB, Rider JE, Hoffman NG, Fowlkes DM, Quillam LA, Kay BK (1996) Distinct ligand preferences of Src homology 3 domains from Src, Yes, Abl, Cortactin, p53bp2, PLCgamma, Crk, and Grb2. Proc. Natl. Acad. Sci. USA 93:1540-1544.

36. Brown T, Brown N, Stollar EJ (2018) Most yeast SH3 domains bind peptide targets with high intrinsic specificity. PLoS One 13:e0193128.

37. Feng S, Kasahara C, Rickles RJ, Schreiber SL (1995) Specific interactions outside the prolinerich core of two classes of Src homology 3 ligands. Proc. Natl. Acad. Sci. USA 92:12408-12415. 38. Bentley ML, Gaweska H, Kielec JM, McCafferty DG (2007) Engineering the substrate specificity of Staphylococcus aureus Sortase A. The beta6/beta7 loop from SrtB confers NPQTN recognition to SrtA. J. Biol. Chem. 282:6571-6581.

39. Wójcik M, Szala K, van Merkerk R, Quax WJ, Boersma YL (2020) Engineering the specificity of Streptococcus pyogenes sortase A by loop grafting. Proteins 88:1394-1400.

40. Biswas T, Misra A, Das S, Yadav P, Ramakumar S, Roy R (2020) Interrogation of 3D-swapped structure and functional attributes of quintessential Sortase A from Streptococcus pneumoniae. Biochem. J.

41. Misra A, Biswas T, Das S, Marathe U, Sehgal D, Roy RP, Suryanarayanarao R (2011) Crystallization and preliminary X-ray diffraction studies of sortase A from Streptococcus pneumoniae. Acta Crystallogr. Sect. F, Struct. Biol. Cryst. Commun. 67:1195-1198.

42. Huang X, Aulabaugh A, Ding W, Kapoor B, Alksne L, Tabei K, Ellestad G (2003) Kinetic mechanism of Staphylococcus aureus sortase SrtA. Biochemistry 42:11307-11315.

43. Race PR, Bentley ML, Melvin JA, Crow A, Hughes RK, Smith WD, Sessions RB, Kehoe MA, McCafferty DG, Banfield MJ (2009) Crystal structure of Streptococcus pyogenes sortase A: implications for sortase mechanism. J. Biol. Chem. 284:6924-6933.

44. Khare B, Samal A, Vengadesan K, Rajashankar KR, Ma X, Huang IH, Ton-That H, Narayana SVL (2010) Preliminary crystallographic study of the Streptococcus agalactiae sortases, sortase A and sortase C1. Acta Crystallogr. Sect. F, Struct. Biol. Cryst. Commun. 66:1096-1100.

45. Khare B, Krishnan V, Rajashankar KR, I-Hsiu H, Xin M, Ton-That H, Narayana SV (2011) Structural differences between the Streptococcus agalactiae housekeeping and pilus-specific sortases: SrtA and SrtC1. PLoS One 6:e22995.

46. Schmohl L, Bierlmeier J, von Kügelgen N, Kurz L, Reis P, Barthels F, Mach P, Schutkowski M, Freund C, Schwarzer D (2017) Identification of sortase substrates by specificity profiling. Bioorg. Med. Chem. 25:5002-5007.

47. Kruger RG, Dostal P, McCafferty DG (2004) Development of a high-performance liquid chromatography assay and revision of kinetic parameters for the Staphylococcus aureus sortase transpeptidase SrtA. Anal. Biochem. 326:42-48.

48. Zong Y, Bice TW, Ton-That H, Schneewind O, Narayana SVL (2004) Crystal structures of Staphylococcus aureus sortase A and its substrate complex. J. Biol. Chem. 279:31383-31389.

49. Li H, Chen Y, Zhang B, Niu X, Song M, Luo Z, Lu G, Liu B, Zhao X, Wang J, et al. (2016) Inhibition of sortase A by chalcone prevents Listeria monocytogenes infection. Biochem. Pharmacol. 106:19-29.

50. UniProt Consortium (2012) Reorganizing the protein space at the Universal Protein Resource (UniProt). Nucleic Acids Res. 40:D71-5.

51. Magrane M, Consortium U (2011) UniProt Knowledgebase: a hub of integrated protein data. Database (Oxford) 2011:bar009.

52. Wu Q, Ploegh HL, Truttmann MC (2017) Hepta-Mutant Staphylococcus aureus Sortase A (SrtA7m) as a Tool for in Vivo Protein Labeling in Caenorhabditis elegans. ACS Chem. Biol. 
Gao and Johnson et al.

12:664-673.

53. Hess GT, Guimaraes CP, Spooner E, Ploegh HL, Belcher AM (2013) Orthogonal labeling of M13 minor capsid proteins with DNA to self-assemble end-to-end multiphage structures. ACS Synth. Biol. 2:490-496.

54. Guimaraes CP, Witte MD, Theile CS, Bozkurt G, Kundrat L, Blom AEM, Ploegh HL (2013) Site-specific C-terminal and internal loop labeling of proteins using sortase-mediated reactions. Nat. Protoc. 8:1787-1799.

55. Wilkins MR, Gasteiger E, Bairoch A, Sanchez JC, Williams KL, Appel RD, Hochstrasser DF (1999) Protein identification and analysis tools in the ExPASy server. Methods Mol. Biol. 112:531-552.

56. Kabsch W (2010) XDS. Acta Crystallogr. Sect. D, Biol. Crystallogr. 66:125-132.

57. Kabsch W (2010) Integration, scaling, space-group assignment and post-refinement. Acta Crystallogr. Sect. D, Biol. Crystallogr. 66:133-144.

58. Chen VB, Arendall WB, Headd JJ, Keedy DA, Immormino RM, Kapral GJ, Murray LW, Richardson JS, Richardson DC (2010) MolProbity: all-atom structure validation for macromolecular crystallography. Acta Crystallogr. Sect. D, Biol. Crystallogr. 66:12-21.

59. Emsley P, Lohkamp B, Scott WG, Cowtan K (2010) Features and development of Coot. Acta Crystallogr. Sect. D, Biol. Crystallogr. 66:486-501.

60. Adams PD, Afonine PV, Bunkóczi G, Chen VB, Davis IW, Echols N, Headd JJ, Hung L-W, Kapral GJ, Grosse-Kunstleve RW, et al. (2010) PHENIX: a comprehensive Python-based system for macromolecular structure solution. Acta Crystallogr. Sect. D, Biol. Crystallogr. 66:213-221.

61. Notredame C, Higgins DG, Heringa J (2000) T-Coffee: A novel method for fast and accurate multiple sequence alignment. J. Mol. Biol. 302:205-217.

62. Altschul SF, Gish W, Miller W, Myers EW, Lipman DJ (1990) Basic local alignment search tool. J. Mol. Biol. 215:403-410.

63. Clamp M, Cuff J, Searle SM, Barton GJ (2004) The Jalview Java alignment editor. Bioinformatics 20:426-427.

64. Crooks GE, Hon G, Chandonia JM, Brenner SE (2004) WebLogo: a sequence logo generator. Genome Res. 14:1188-1190. 
Gao and Johnson et al.

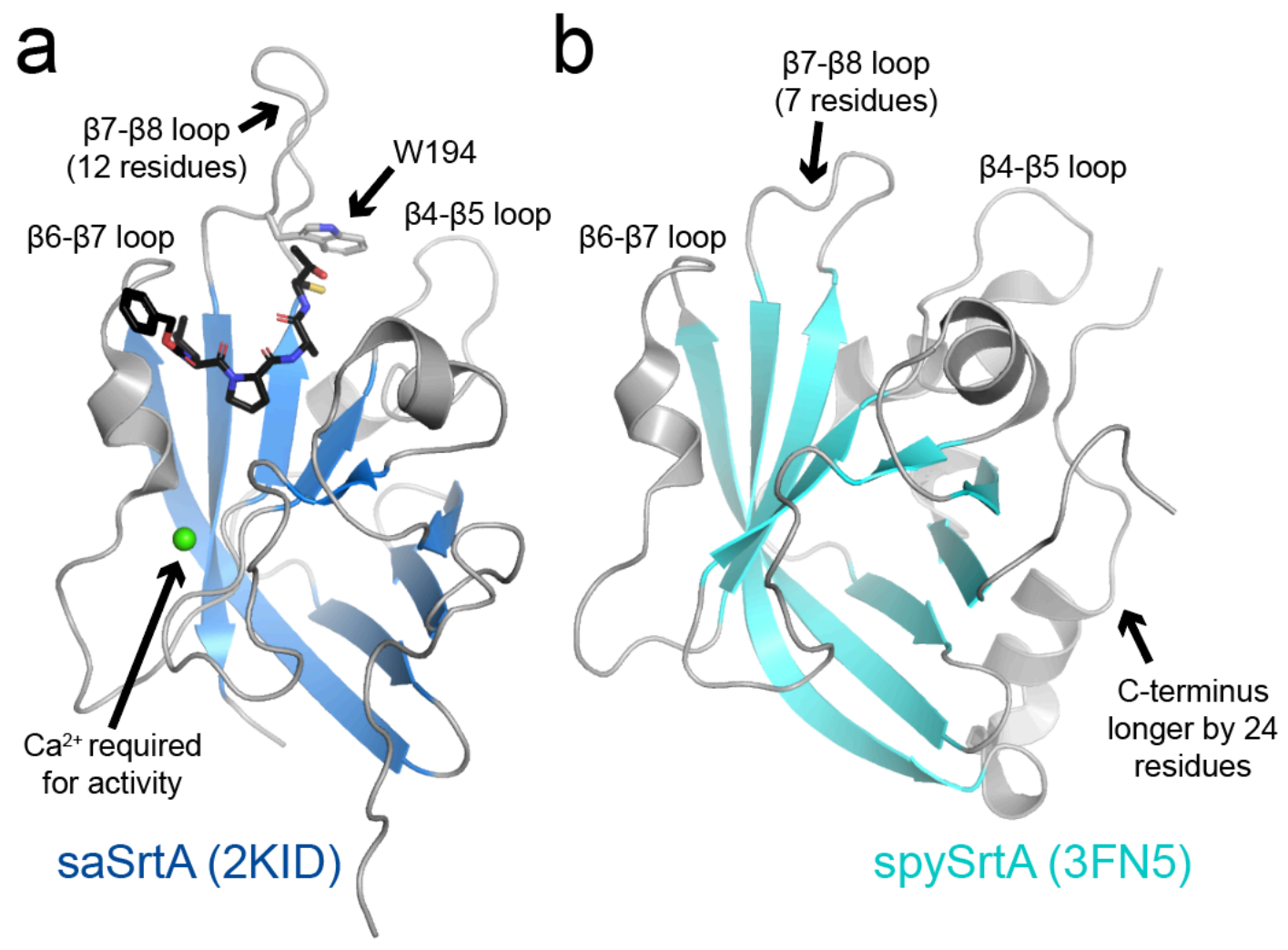

Figure 1. Differences between Staphylococcus and Streptococcus SrtA proteins. The SrtA proteins are in cartoon representation, with the conserved 8-stranded antiparallel $\beta$-sheet that defines the sortase fold colored as labeled. S. aureus SrtA (saSrtA, PDB ID 2KID) is bound to the peptidomimetic, LPAT*, in black sticks and colored by heteroatom $(\mathrm{O}=\mathrm{red}, \mathrm{N}=$ blue, $\mathrm{S}=$ yellow $)$, (a). The arrows indicate differences with $S$. pyogenes SrtA (spySrtA, 3FN5) (b). Specifically, $\mathrm{Ca}^{2+}$ is required for saSrtA activity, the $\beta 7-\beta 8$ loop contains an additional 5 residues and contains a Trp (W194) that dramatically affects activity, and the spySrtA protein is 24 residues longer than saSrtA. 
Gao and Johnson et al.

a

SPSTA 81 AVLT SOWDAOKLPVI GG I A I PELEMNLP I FKGLDNVNLFYGAG sagSitA 79 SILSAOTKSHNLPVIGGIAIPDVEINLPIFKGLGNTELSYGAG SpySitA 81 SVLQAOMAAQQL PVIGGIAIPELGINLPIFKGLGNTELIYGAG

SpSrtA 124 TMKREQVMG-EGNYSLASHH I FGVDNANKMLFSPLDNAKNGMK sagSrtA 122 TMKENQIMCGPNN YALA S H HVF GLTCS SKML F S P LEHAKKGMK SPYSrtA 124 TMKEEQVMGGENN Y S LASHH I FGITGS SQMLF S P LERAONGMS

SpSrtA 166 I YLTDKNKVYTYETREVKRVTPDRVDEVDDRDGVNGITLV!CE SAgSIA 165 VYLTDKSKVYTYTITEISKVTPEHVEVIDDTPGKSOLTLV!CT SpYSrtA 167 I Y LTDKEKIYEYIIKDVF TVAPERVDVIDDTAGLKEVTLV!CC

SpSrtA 209 DLAATERI I VKGDLKETKDYSOT S DEI ITAF NOPYKOFY SagSrtA 208 DPEATER I I VHAEIEKTGEFSTADESI LKAFSKKYNQINL SPYSrtA 210 DIEATERI I VKGELKTEYDFDKAPADVLKAFNHSYNQVST

$\mathrm{b}$

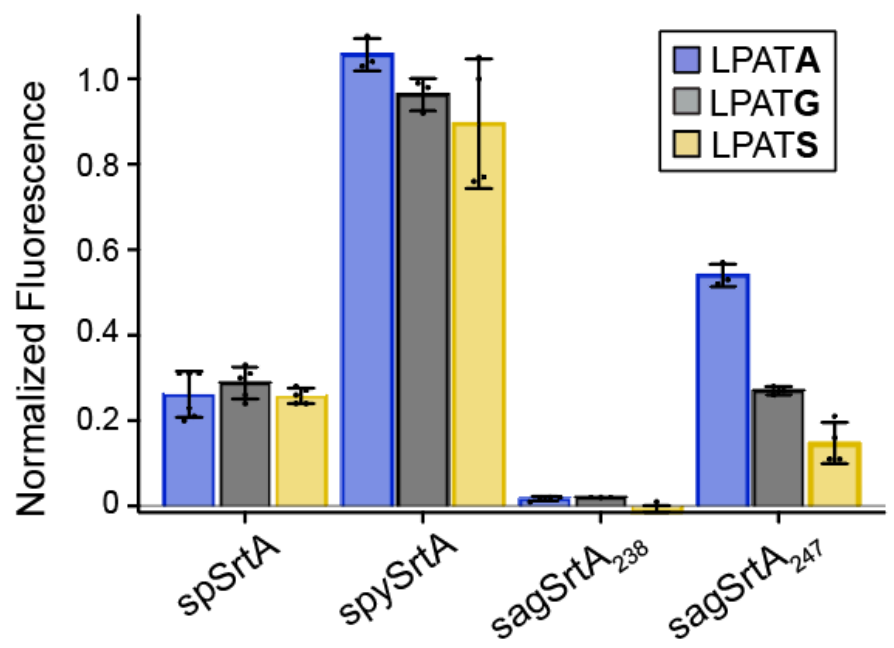

Figure 2. Biochemical characteristics of SrtA enzymes from S. agalactiae and S. pyogenes. (a) Sequence alignment of the extracellular regions of the $S$. pneumoniae SrtA (spSrtA), $S$. agalactiae SrtA (sagSrtA) and S. pyogenes SrtA (spySrtA) proteins. Sequences were aligned using T-coffee and visualized with Boxshade. The $\beta 7-\beta 8$ loop residues are indicated with a red box. (b) Comparison of substrate selectivity for wild-type spSrtA, spySrtA, sagSrtA238 (the crystallized construct reported in PDB ID 3RCC), and spySrtA 247. Substrate cleavage was monitored via an increase in fluorescence at $420 \mathrm{~nm}$ from reactions of the fluorophore-quencher probes AbzLPATGG-K(Dnp), Abz-LPATAG-K(Dnp), and Abz-LPATSG-K(Dnp) (represented as LPATG, LPATA, and LPATS) in the presence of excess hydroxylamine. Bar graphs represent mean normalized fluorescence ( \pm standard deviation) from at least three independent experiments at the $2 \mathrm{~h}$ reaction timepoint, as compared to saSrtA and the peptide LPATG. The spSrtA data was previously published and is shown for comparison. ${ }^{12}$ Averaged assay values and standard deviations for spySrtA, sagSrtA 238 , and sagSrtA 247 are in Table S1. 
Gao and Johnson et al.
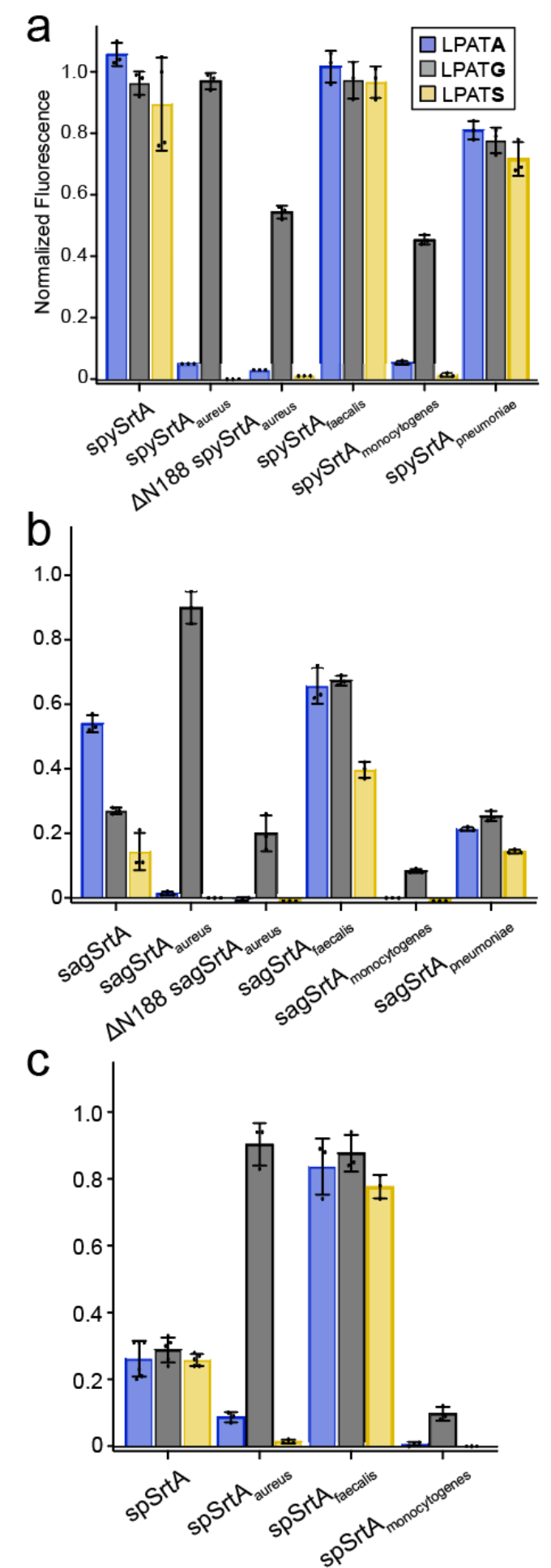

Figure 3. Enzyme assays of $\beta 7-\beta 8$ loop chimeras of spySrtA, $\operatorname{sagSrtA}$, and $\operatorname{spSrtA}$. Comparison of substrate selectivity for $\beta 7-\beta 8$ loop chimeras of (a) spySrtA, (b) sagSrtA, and (c) spSrtA proteins. Assays were run and data was collected as in Figure 2. Averaged assay values and standard deviations for spySrtA and sagSrtA variants are in Table S1. The spSrtA data was previously published and is shown for comparison. ${ }^{12}$ 
Gao and Johnson et al.

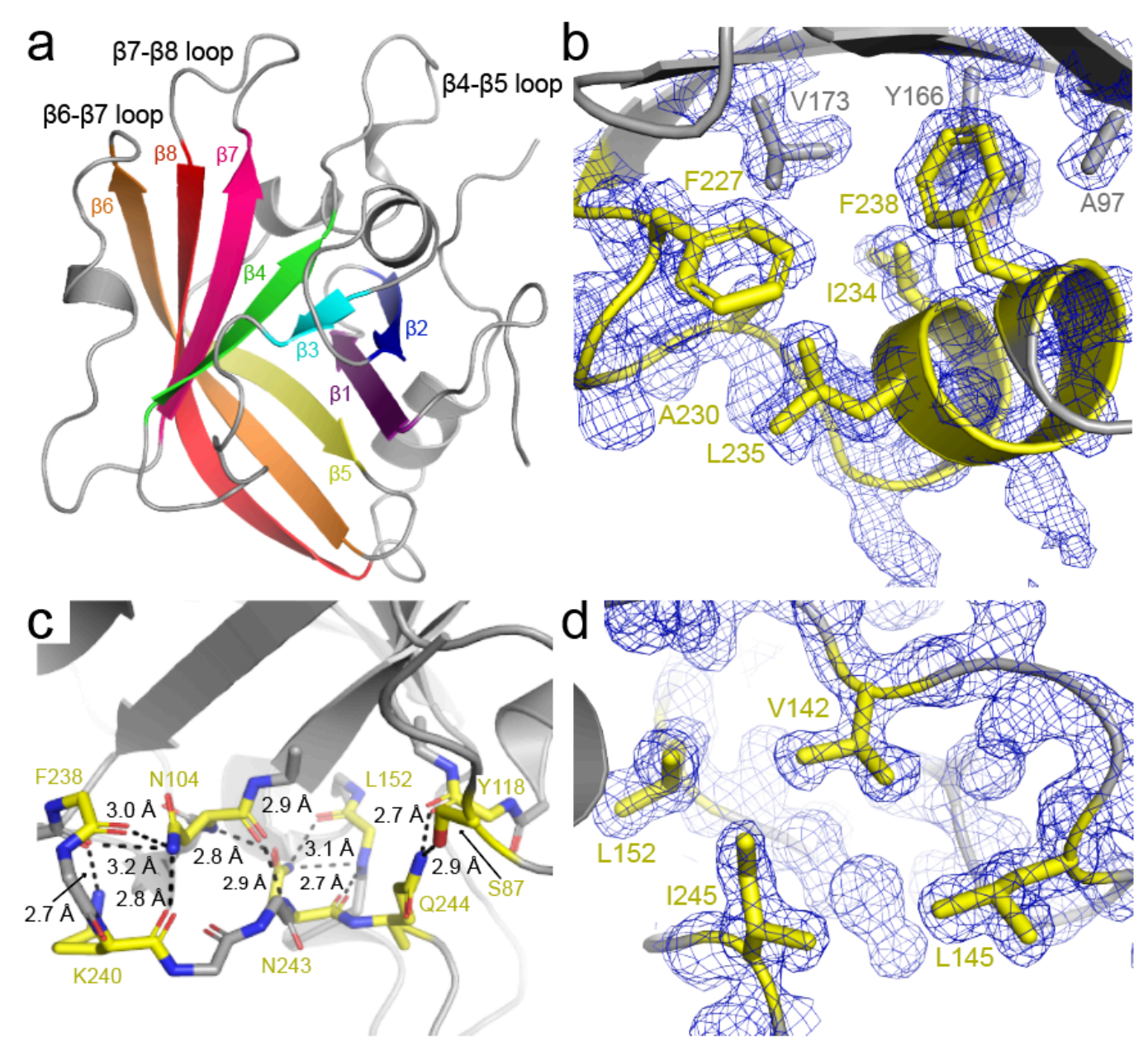

Figure 4. Structural characteristics of sagSrtA247 (a) $\mathrm{SagSrtA}_{247}$ adopts the conserved sortase fold. The protein is in gray cartoon, with $\beta$-strands numbered and colored as labeled. (b) C-terminal residues in sagSrtA 247 that were in the construct previously crystallized, but were not resolved in the structure, make intra-protein hydrophobic interactions. Unresolved residues from PDB ID $3 \mathrm{RCC}$ are in yellow, with other sagSrtA $\mathrm{A}_{247}$ residues in gray. Residues involved in the interaction are labeled and their side chains are shown as sticks. The electron density in this region is also shown, the $2 F_{\mathrm{o}}-F_{\mathrm{c}}$ map is rendered at $1 \sigma$. (c) C-terminal residues not included in the previously crystallized construct make several interactions in sagSrtA 247 . All distances are labeled and residues involved in contacts (including main chain of residues 238-244) are shown as sticks and colored by heteroatom $(\mathrm{C}=$ yellow, $\mathrm{O}=\mathrm{red}, \mathrm{N}=$ blue). Side chain atoms are shown when involved in the interaction, otherwise they are omitted for visual clarity. (d) The hydrophobic interaction involving I245 is shown, all residues are colored and the electron density is as in (b). 
Gao and Johnson et al.
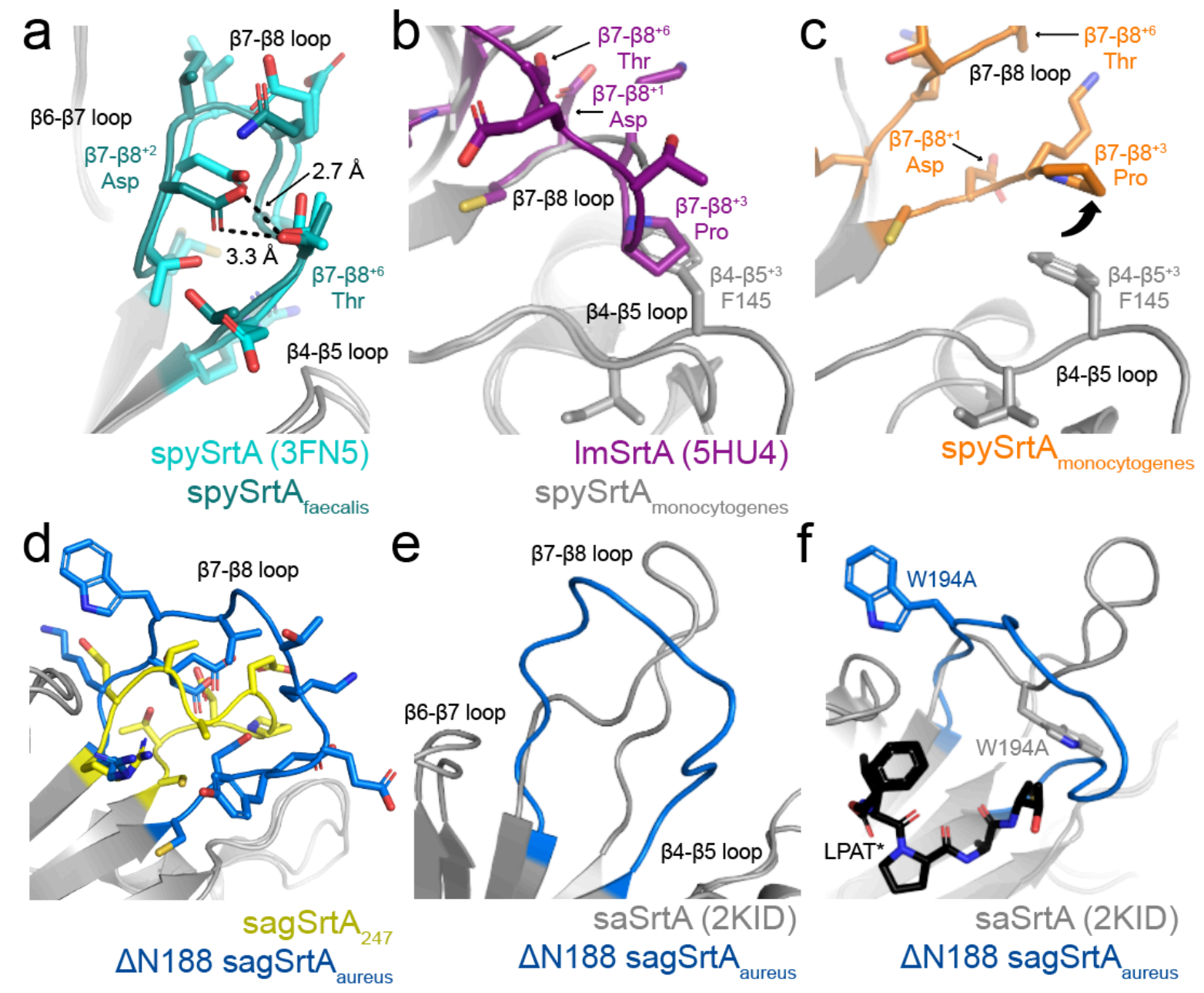

Figure 5. Structural characteristics of sagSrtA and spySrtA $\beta 7-\beta 8$ loop chimeras. In all, relevant residues are colored by structure as labeled and when represented, side chains are shown as sticks and colored by heteroatom $(\mathrm{O}=\mathrm{red}, \mathrm{N}=$ blue $)$. Other residues are shown as gray cartoon. (a) The intraloop hydrogen bond is conserved between spySrtA (PDB ID 3FN5) and spySrtA faecalis. (b) The $\beta 7-\beta 8^{+3}$ Pro in lmSrtA (5HU4) occupies the same position as the $\beta 4-\beta 5^{+3}$ Phe of

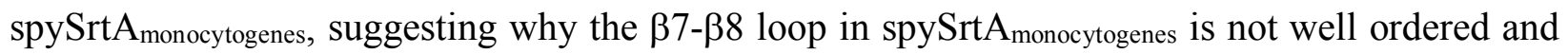
this variant is not as active. Furthermore, the intraloop hydrogen bond is not maintained in

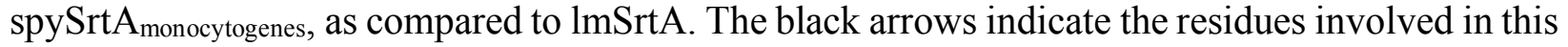
interaction in $1 \mathrm{mSrtA}$. (c) The $\beta 7-\beta 8^{+3}$ Pro in spySrtA monocytogenes is translated up, as compared to that of $1 \mathrm{mSrtA}$ in (b). The black arrows show the relevant residues for the intra-loop hydrogen bond that is not conserved, as compared to (b). (d-f) Comparison of the $\Delta$ N188 sagSrtA $\mathrm{A}_{\text {aureus }} \beta 7-\beta 8$ loop with sagSrtA 247 (d) and saSrtA (2KID, e-f). In (f), the LPAT* peptidomimetic is shown as black sticks and colored by heteroatom. The different positions of W194 are labeled. 
Gao and Johnson et al.

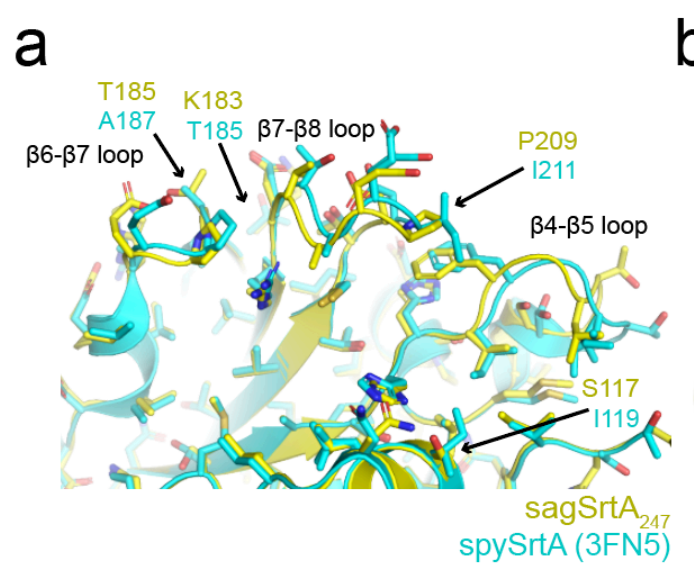

d

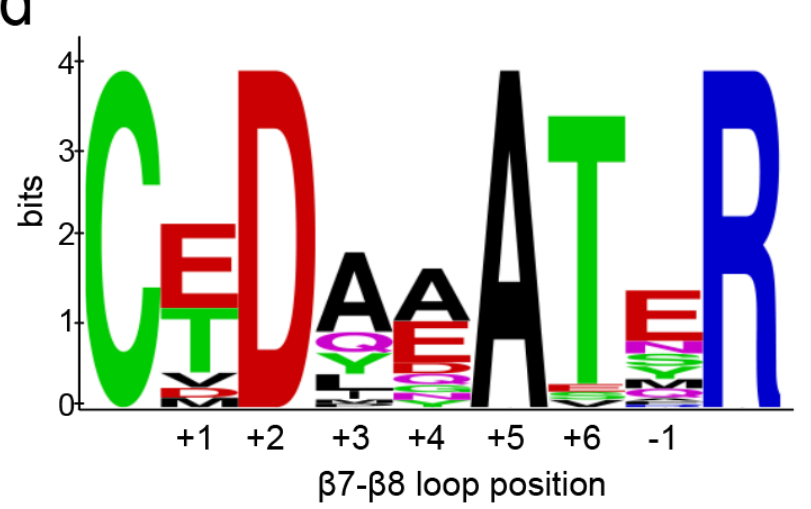

b

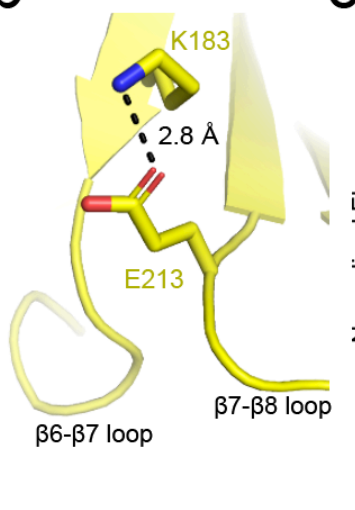

C

e

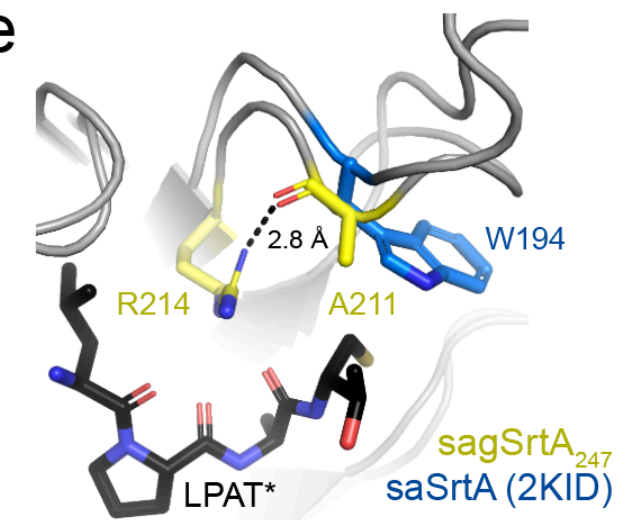

Figure 6. Residues in the $\beta 7-\beta 8$ loop of Streptococcus proteins that regulate enzyme function. (a) Although the peptide-binding pockets of sagSrtA and spySrtA are well conserved, there are four non-conservative amino acid differences, as labeled. Both proteins are shown in cartoon representation, with side chains as sticks and colored by heteroatom. The $\beta 4-\beta 5, \beta 6-\beta 7$, and $\beta 7-\beta 8$ loops are also labeled. (b) The side chain of $\beta 7-\beta 8^{-1}$ E213 interacts with that of $\beta 6^{-2} \mathrm{~K} 183$ in sagSrtA247. This is an interaction that negatively affects enzyme activity for spSrtA, as previously reported, and which spySrtA does not share. ${ }^{12}$ (c) Mutation of the $\beta 7-\beta 8^{+3}$ residue in sagSrtA from Pro to Ile, as in spySrtA, increases activity. However, the converse mutation in spySrtA, from Ile to Pro, has little to no effect. Assays were run and data was collected as in Figures 2-3. Averaged assay values and standard deviations are in Table S1. (d) WebLogo analysis of 37 Streptococcus SrtA proteins from the UniProt database. All sequences are in Table S2. (e) The absolutely conserved $\beta 7-\beta 8^{+5}$ Ala in the sequences in (d) is stereochemically located in the same position as the W194 residue in saSrtA (2KID). A hydrogen bond between the A211 carbonyl and guanidinium group of the catalytic R214 is labeled. 